\begin{tabular}{|c|c|}
\hline Title & Picosecond time resolved fluorescence spectroscopy of hematoporphyrin derivative \\
\hline Author(s) & Y amashita, Mikio; Nomura, Masahide; Kobay ashi, Shunsuke; Sato, T akuzo; A izawa, Katsuo \\
\hline Citation & $\begin{array}{l}\text { IEEE Journal of Quantum Electronics, 20(12), 1363-1369 } \\
\text { https://doi.org/10.1109/QQE.1984.1072318 }\end{array}$ \\
\hline Issue Date & 198412 \\
\hline Doc URL & http:/hdl.handle.net/2115/45318 \\
\hline Rights & $\begin{array}{l}\text { C 1984 IEEE. Personal use of this material is permitted. However, permission to reprint/republish this material for } \\
\text { advertising or promotional purposes or for creating new collective works for resale or redistribution to servers or lists, } \\
\text { or to reuse any copyrighted component of this work in other works must be obtained from the IEEE. }\end{array}$ \\
\hline Type & article \\
\hline File Information & JQE20-12_1363-1369.pdf \\
\hline
\end{tabular}

Instructions for use 


\title{
Picosecond Time-Resolved Fluorescence Spectroscopy of Hematoporphyrin Derivative
}

\author{
MIKIO YAMASHITA, MASAHIDE NOMURA, SHUNSUKE KOBAYASHI, \\ TAKUZO SATO, AND KATSUO AIZAWA
}

(Invited Paper)

\begin{abstract}
The picosecond time-resolved fluorescence decays $I(t)$ and spectra $I(\lambda, t)$ for hematoporphyrin derivative (HPD) in a phosphate buffer saline aqueous solution at different concentrations $(8.4 \times$ $10^{-6} \sim 8.4 \times 10^{-3} \mathrm{M}$ ) are measured by a two-dimensional synchrosean streak camera with a mode-locked $\mathrm{CW}$ dye laser, and a new emission band (which we call the $Y$-band) is found at high concentration. It is shown that the fluorescence decays composed of fast and slow components at high concentration are due to the $Y$-band (120 ps lifetime) from head-to-tail aggregates including equilibrium dimer and stable dimer, and the usual band ( 3.6 ns lifetime) from monomer, respectively, and the latter band is dynamically quenched by the Forster type resonance energy transfer from the monomer to the aggregate. Furthermore, the measurement of static fluorescence spectra from human gastric cancers and the surrounding in vivo after HPD injection shows that a band corresponding to the $Y$-band from the aggregate appears at only the cancerous cells.
\end{abstract}

\section{INTRODUCTION}

$\mathrm{R}$ ECENTLY, it has been demonstrated that photoradiation therapy with the use of hematoporphyrin derivative (HPD) activated by laser light at a wavelength of $620-640 \mathrm{~nm}$ is an effective treatment of various types of cancers [1]- [3] . There has been an extensive investigation of the behavior of HPD from physical, photochemical, and biological aspects [4] . Many of the mechanisms of energy relaxations and photochemical reactions in the course of the treatment are not yet made clear. In order to reveal the mechanisms, there are the following four subjects to be investigated: 1) elucidation for mechanisms of decay kinetics and energy transfer from the excited state after absorbing the laser light, 2) identification of intermediate states and products in the processes of photochemical reactions and determination of their kinetic rates, 3) identification of target biomolecules which are finally chemically changed and subsequently do not enable cancerous cells to live, and 4) elucidation of the reason why HPD accumulates selectively in cancerous cells.

According to an analysis by Dougherty's group, HPD consists of a mixture of hematoporphyrin IX (HPIX), hydroxyethylvinyl-deuteroporphyrin IX (HVDPIX), protoporphyrin

Manuscript received January 12, 1984; revised July 16, 1984.

M. Yamashita, M. Nomura, S. Kobayashi, and T. Sato are with the Laser Research Section, Radio- and Opto-Electronics Division, Electrotechnical Laboratory, Ibaraki 305 , Tokyo, Japan.

K. Aizawa is with the Department of Physiology, Tokyo Medical College, Tokyo 160, Japan.
IX (PPIX), and their aggregates, including ether-bonded dimer of HVDPIX and HPIX [4], [5]. On the other hand, it is already known from static spectroscopic studies that HPIX, DPIX, and PPIX are easily dimerized in the equilibrium with monomer in a neutral aqueous solution [6]-[10]. Therefore, it is deduced that in the HPD solution two types of dimers exist, one is chemically bonded and stable, and the other is kinetically in the equilibrium with the monomer and the concentration dependent. For prevention of confusion we define aggregates in this paper as follows: the aggregates mean ether-bonded dimers (composite molecules, $S$ dimers) and dissociable aggregates ( $D$ aggregates). The latter are weak coupling dimers ( $E$ dimers), timers, and oligomers such as van der Waals coupling aggregates, which are in the equilibrium with molecular units and the concentration dependent. More recently from clinical studies on the relation between HPD components and treatment efficacy, it has been pointed out that the aggregates are essential to the effective treatment of cancer by photoradiation therapy [4], [5], [11], [12]. This is thought to be due to the point that the selective accumulation of HPD involves the properties of aggregation of HPD components.

Recent pulse-laser techniques have made it possible to resolve excited state decay kinetics for a HPD solution. The fluorescence decay kinetics from the first excited singlet state has been investigated in the time region of nanoseconds by Milan's group [13] and in picoseconds by the present authors [14] and Rochester's group [15]. Consequently, it has been found that a fluorescence decaying curve of HPD in a phosphate buffer saline aqueous solution (PBS solution) shows two components of fast (less than a few hundred picoseconds) and slow (more than several nanoseconds) decays [14], [15]. The fluorescence decay increases when the HPD concentration decreases. It is generally thought that the fast and slow decay components are due to aggregates and monomers, respectively [15]. However, no direct evidence for the existence of a fast emission band from aggregates, which is clearly distinguished from a slow fluorescence band from monomers, has yet been obtained. It is generally known that the fluorescence from aggregates of organic molecules rapidly decays in the picosecond time-region [16], [17]. In this paper, we report the first investigation of the picosecond time-dependent fluorescence spectra $I(\lambda, t)$ from HPD in a PBS solution by using a two-dimensional synchroscan streak camera method. A new 
emission band (which we call the $Y$-band) due to the aggregates is found. In addition, mechanisms of the fluorescence relaxation for the HPD solution are quantitatively discussed, including the effect of resonance energy transfer from monomers to aggregates. The result of the direct measurement of the static fluorescence spectra for human gastric cancers after HPD injection in vivo is compared with the dynamic fluorescence spectra, and it is shown that a third emission band corresponding to the $Y$-band appears only at the cancerous cells after injection with HPD.

\section{EXPERIMENTAL}

The HPD solution of $5 \mathrm{mg} / \mathrm{ml}\left(8.4 \times 10^{-3} \mathrm{M}\right)$ concentration was offered from Dougherty's group [12] and diluted to desired concentrations with a PBS solution $(0.02 \mathrm{M}, \mathrm{pH}=7.2)$. The diluted HPD solution in a quartz cell was circulated to avoid any effects from photodegradation. The experimental apparatus for measuring picosecond time-dependent fluorescence spectra from the HPD solution is improved in comparison with the one we described in previous papers [14], [18]. A Jovin Yvon HR320 polychromator was attached between a sample optics and a Hamamatsu C1587 synchroscan streak camera. For data recording and processing, a Princeton Applied Research OMA II optical multichannel analyzer (two-dimensional) with a SIT visicon of high dynamic range $\left(1.6 \times 10^{4}\right)$ and sensitivity (20 photons/count) was used. The sensitivity of the synchroscan streak camera was also improved by $\sim 50$ times as high as the previous one by replacing a microchannel plate incorporated in a streak tube. A tunable synchronously mode-locked $\mathrm{CW}$ dye (R6G) laser which was constructed by the present authors generates continuous trains of pulses at $82 \mathrm{MHz}$ with an average power of $\sim 30 \mathrm{~mW}$ and a duration of less than 5 ps at $570 \mathrm{~nm}$. A $570 \mathrm{~nm}$ laser light was employed as an excitation source to avoid photodegradation due to irradiation around $400 \mathrm{~nm}$ of the absorption peak. The overall time-resolution of the system was examined by measuring the duration of pulses from the dye laser. The recorded pulse duration at the present operational conditions was 19 ps at the accumulation of $\sim 10^{9}$ pulses. The reabsorption effect of the fluorescence at high concentration was carefully avoided by measuring the front fluorescence near the surface of the sample cell pumped at some angle in respect to its surface.

All absorption spectra for HPD in the PBS solution were carefully measured on a Hitachi Model 340 recording spectrophotometer over a wide range of concentration $\left(2.5 \times 10^{-8}\right.$ $8.4 \times 10^{-3} \mathrm{M}$ ). This was made possible by the use of cuvettes of different path lengths $(0.1,1.0,10.0$, and $100 \mathrm{~mm})$ and by calibrations of background spectra of their cuvettes containing the PBS solution without HPD. Measurements of fluorescence spectra and fluorescence excitation spectra were made on a Hitachi Model 850 fluorescence spectrophotometer. The fluorescence spectra were taken at the excitation wavelengths of 390,515 , or $570 \mathrm{~nm}$. The excitation spectra were measured at 615 or $680 \mathrm{~nm}$.

\section{Results AND Discussion}

\section{A. Steady-State Absorption and Fluorescence Excitation Spectra}

Absorption spectra of HPD in the PBS solution measured over the concentration range from $2.5 \times 10^{-8}-8.4 \times 10^{-3} \mathrm{M}$

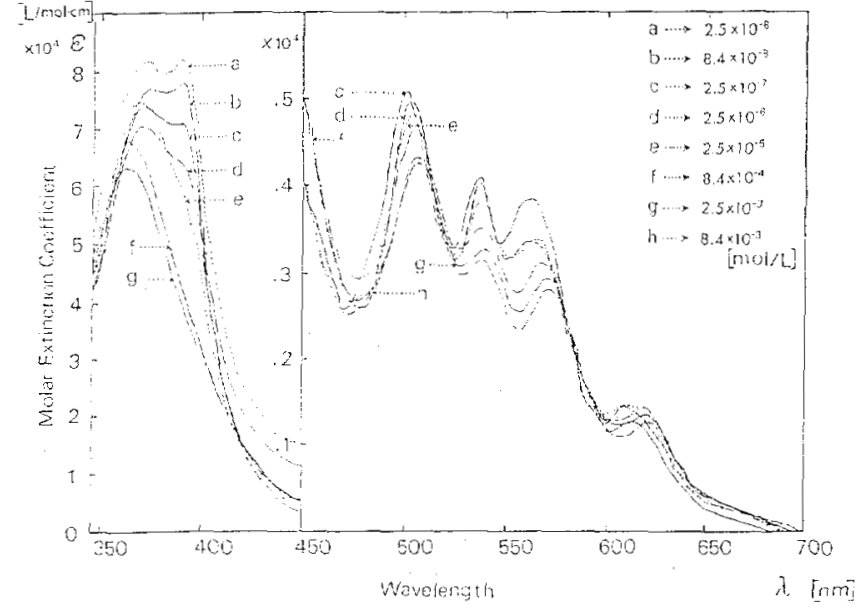

Fig. 1. Absorption spectra of different concentrations of HPD in the PBS solution: (a) $2.5 \times 10^{-8}$, (b) $8.4 \times 10^{-8}$, (c) $2.5 \times 10^{-7}$, (d) $2.5 \times 10^{-6}$, (e) $2.5 \times 10^{-5}$, (f) $8.4 \times 10^{-4}$, (g) $2.5 \times 10^{-3}$, and (h) $8.4 \times 10^{-3} \mathrm{M}$.

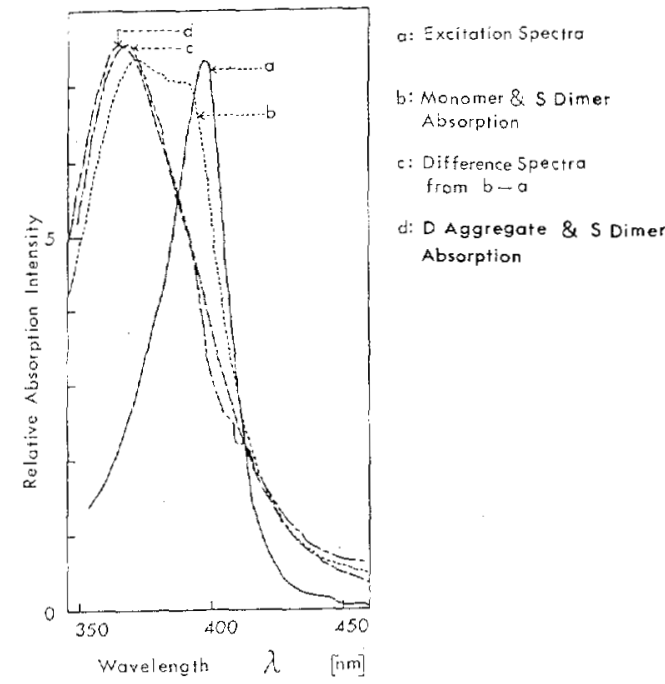

Fig. 2. (a) (- $\longrightarrow$ ) and (b) (----) are fluorescence excitation (corresponding to monomer absorption) spectrum and absorption spectrum at the diluted concentration (corresponding to absorption spectrum of monomer and $S$ dimer), respectively. (c) (- . - - ) is the difference spectrum subtracted from (b) by (a), corresponding to the absorption spectra of $S$ dimer. (d) (- - ) is the absorption spectrum at high concentration corresponding to absorption spectra of $D$ aggregate and $S$ dimer.

are shown in Fig. 1. It is seen from Fig. 1 that with the increase of the concentration the molar extinction coefficient decreases, the spectral shape of the Soret band changes from two peaks at 372 and $390 \mathrm{~nm}$ to a single peak at $363 \mathrm{~nm}$, and a red-shift of four peaks in the $Q$-band occurs. This behavior is very similar to the change from the absorption spectra of the monomer to those of the $E$ dimer of HPIX, DPIX, and PPIX in an aqueous solution [7]-[10], except that the peak at the shorter wavelength of the Soret band still remains at the most diluted concentration. This suggests that in the HPD solution at high concentration the $D$ aggregate including mainly the $E$ dimer exists, while at the diluted concentration, in addition to the monomer, another molecular species independent of the concentration exists. In order to confirm the latter part of this suggestion, we measured how the relative intensity of the fluorescence peaks at 615 or $680 \mathrm{~nm}$ depends on the wavelength of the excitation light. A typical excitation spectrum independent of the concentration is shown in Fig. 2(a). 


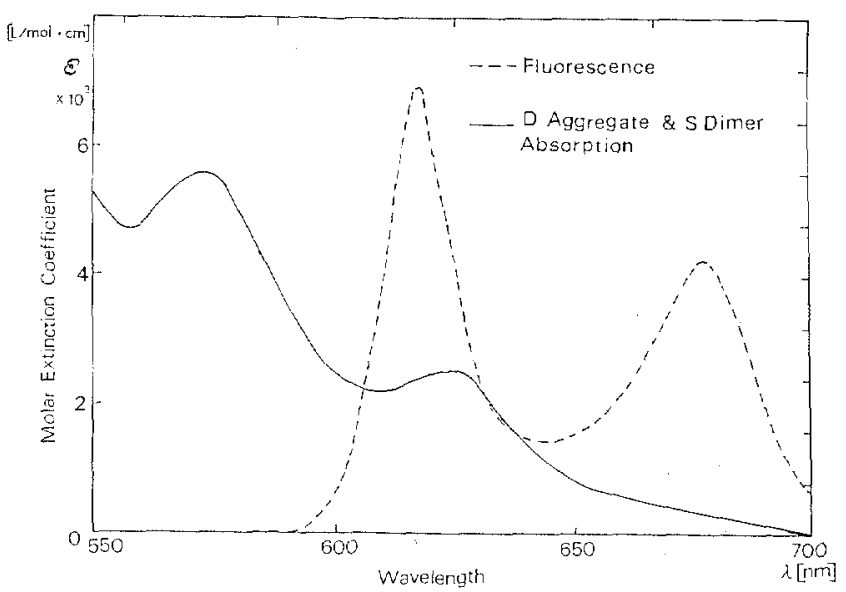

Fig. 3. Overlap of fluorescence spectra $(\cdots)$ and absorption spectra of $D$ aggregate and $S$ dimer ( $\longrightarrow$ ).

Favorable comparison of this spectrum with the absorption spectrum of the monomer of HPIX and DPIX already known [7] - [9] indicates that both the spectra are almost the same. This means that the monomer of HPD emits the fluorescence having two peaks of 615 and $680 \mathrm{~nm}$ like the monomer of HPIX, DPIX, and PPIX. Fig 2(c) is the difference spectrum, which was subtracted from the absorption spectrum at the diluted concentration [Fig. 2(b)] by the excitation spectrum so as not to become negative. This difference spectrum coincides with the absorption spectrum at high concentration [Fig. 2(d)], as shown in Fig. 2(c) and (d). This fact gives the evidence that the HPD solution contains not only the $D$ aggregate, but also another stable species in addition to the monomer which is independent of the concentration. It is deduced that this corresponds to the recent analytical result that the HPD solution contains ether-bonded dimer ( $S$ dimer) of HVDPIX and HPIX [4]- [5]. Therefore, it seems that the absorption spectrum of the $S$ dimer is similar to that of the $D$ aggregate containing mainly the $E$ dimer.

Furthermore, the spectral overlap between the fluorescence and the absorption was examined as shown in Fig. 3. The fluorescence spectrum from the monomer overlaps the absorption spectrum at the high concentration due to the $D$ aggregate and $S$ dimer over the range between 590 and $700 \mathrm{~nm}$. Therefore, the dynamic fluorescence quenching due to the resonance energy transfer can occur in the HPD solution such as a rhodamine $B$ monomer-dimer system in an aqueous solution [19] - [20]. This dynamic phenomenon will be discussed later.

\section{B. Picosecond Time-Resolved Fluorescence Measurement}

The measured picosecond decays $I(t)$ of the total fluorescence spectra at different concentrations $\left(8.4 \times 10^{-3}, 8.4 \times\right.$ $10^{-4}, 8.4 \times 10^{-5}$, and $8.4 \times 10^{-6} \mathrm{M}$ ) are shown in Fig. 4. From Fig. 4 it is seen that the decays have fast and slow components at high concentration, which agrees with the experimental result of [15], and the amplitude of the fast component decreases with the decrease of the concentration. In order to clarify the mechanism of the fluorescence relaxation, the picosecond time-dependent fluorescence spectra $I(\lambda, t)$ were also measured at the same concentrations. In Fig. 5, the typical time-dependent fluorescence spectra with the corresponding total fluorescence decays at the highest and lowest (1/100) concentrations are shown. It is seen from Fig. 5 that a new (a)

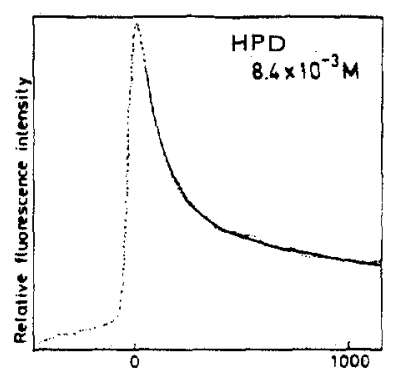

(b)

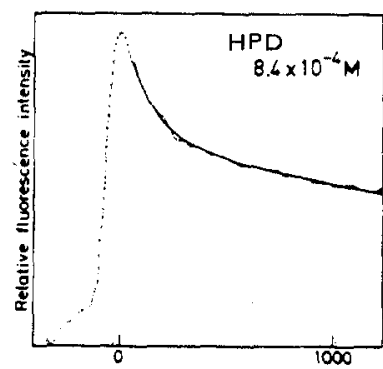

(c)
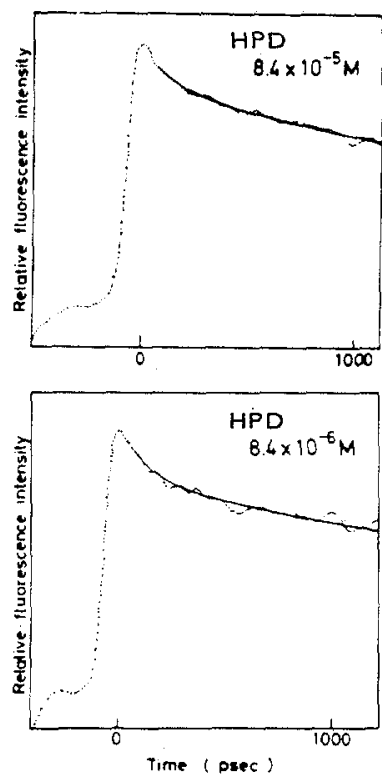

Fig. 4. Picosecond decay $I(t)$ of the total fluorescence for different concentrations of HPD in the PBS solution: (a) $8.4 \times 10^{-3}$, (b) $8.4 \times$ $10^{-4}$, (c) $8.4 \times 10^{-5}$, and (d) $8.4 \times 10^{-6} \mathrm{M}$. The solid lines are numerical fitting curves to the data, as discussed in the text.

emission band (the $Y$-band) around $640 \mathrm{~nm}$ between the usual two bands around 615 and $680 \mathrm{~nm}$ appears in the fast time region for the solution at the highest concentration. The $Y$ band decays faster than a few hundred picoseconds while the usual bands decay slower than several nanoseconds. With decreasing the concentration the $Y$-band is rapidly buried in the usual band. From the comparison between the timedependent spectra and the total fluorescence decays, it is found that the time and concentration variations for the fast component in the decays definitely correspond to the behavior of the $Y$-band. Therefore, it can be concluded that the fast component appearing in the total fluorescence decay is assigned to the $Y$-band emitted from the at least $D$ aggregate. This was also confirmed by measuring the wavelength-resolved fluorescence decays $I(\Delta \lambda, t)$ for the peak of each band, as shown in Fig. 6 .

The same picosecond measurement was also done for the HPD II solution (photofrin II, Oncology Research and Develop- 

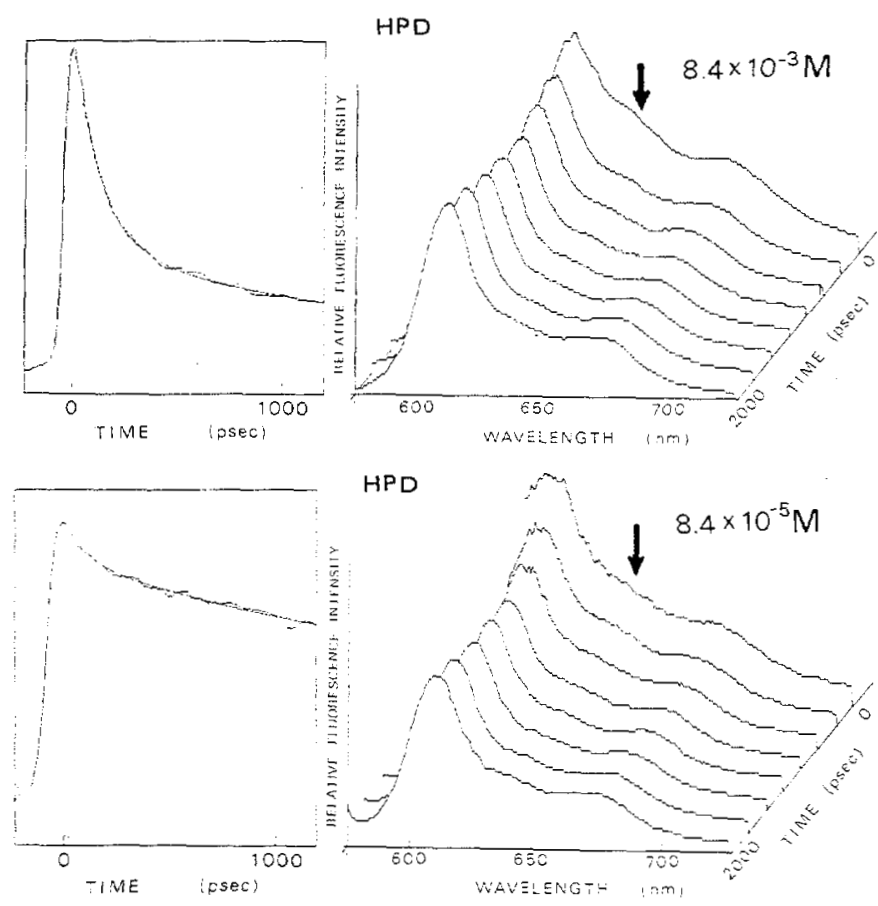

Fig. 5. Picosecond time-dependent fluorescence spectra $I(\lambda, t)$ and corresponding total fluorescence decays $I(t)$ from the HPD solutions at high $\left(8.4 \times 10^{-3} \mathrm{M}\right)$ and low $\left(8.4 \times 10^{-5} \mathrm{M}\right)$ concentrations.

(a)

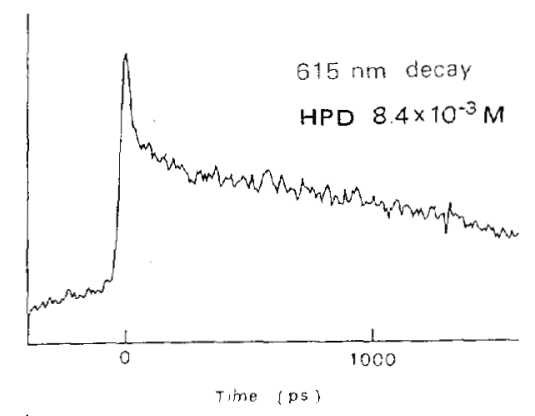

(b)

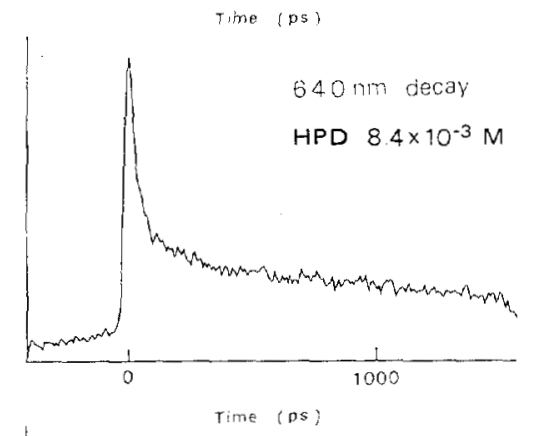

(c)

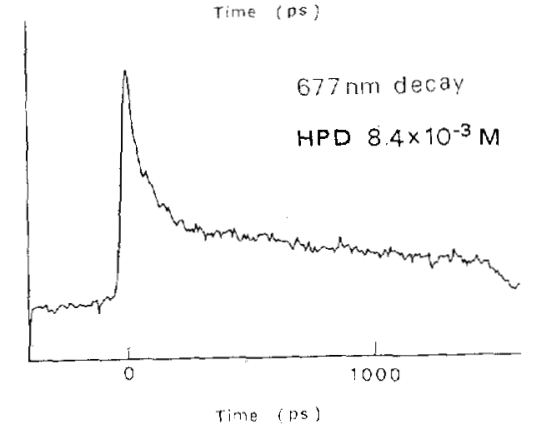

Fig. 6. Wavelength-resolved fluorescence decays $I(\Delta \lambda, t)$ at (a) 615 , (b) 640 , and (c) $677 \mathrm{~nm}$ for the HPD solution at high concentration $\left(8.4 \times 10^{-3} \mathrm{M}\right)$.

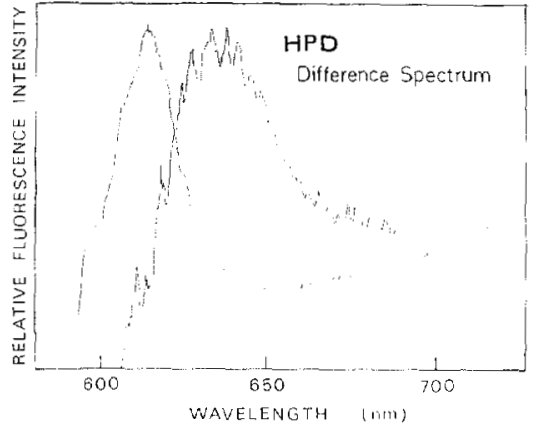

Fig. 7. Difference spectra subtracted from the fluorescence spectra right after pumping (within 300 ps) by the fluorescence spectra long after pumping (6.0 ns passing) for the HPD solution at the high concentrations $\left(8.4 \times 10^{-3} \mathrm{M}\right)$.

ment) [12] in order to examine the effect of the $S$ dimer. The HPD II solution is the effective aggregate fraction separated by gel filtration from the HPD solution and contains much more of the $S$ dimer. It is known from recent clinical studies [3], [4], [11], [12] that the HPD II solution is superior to the HPD solution concerned with the properties of the selective accumulation and the side-effect. The experimental result showed that the above mentioned behaviors of the $Y$-band are more remarkable for the HPD II solution than for the HPD solution. Therefore, it can be said that the $Y$-band is due to not only the $D$ aggregate containing mainly the $E$ dimer, but also the $S$ dimer.

Fig. 7 is the difference spectrum which was subtracted from the fluorescence spectrum right after the excitation pulse $(t=$ $0 \sim 300 \mathrm{ps})$ by that long after the excitation pulse $(t=6.0 \sim$ $6.3 \mathrm{~ns})$ so as not to become negative. From Fig. 7 it is found that the $Y$-band shifts toward the red wavelength in respect to the usual band. It seems that this fact corresponds to the redshift of the $Q$-band in the absorption spectra at high concentration. According to Kasha's theoretical analysis on the energy relaxation from the excited states of the molecular aggregates [16], the face-to-face aggregate (sandwich type) shows the blue-shift relative to the monomer excited state level while the head-to-tail aggregate shows the red-shift. This suggests that both the $D$ aggregate and the $S$ dimer emitting the $Y$ band are the head-to-tail aggregates. It should be noted that the formation of the $S$ dimer necessitates the preformed noncovalent dimer properly aligned [12].

On the basis of the above consideration, we try to describe quantitatively the total fluorescence decay $I(t)$. The decay is the sum of the fast decay of the $Y$-band from the $D$ aggregate and $S$ dimer, and the slow decay of the usual band from the monomer. The latter can be dynamically quenched through the Forster-type resonance energy transfer [20] from the monomer as donor to the $D$ aggregate and $S$ dimer as acceptor since the fluorescence spectrum from the monomer partially overlaps the absorption spectrum at the highest concentration due to the $D$ aggregate and $S$ dimer. Therefore, the total decay $I(t)$ is expressed by the following equation:

$$
I(t)=A_{1} \exp \left[-t / \tau_{1}\right]+A_{2} \exp \left[-\sqrt{\pi} \gamma \sqrt{t / \tau_{2}}-t / \tau_{2}\right]
$$


TABLE I

Fluorescence Relaxation Parameters Obtained from Numerical Fittings for HPD Solutions: Amplitudes of Fast $A_{1}$ and Slow $A_{2}$ Decaying Components, Ratio $\gamma$ of Total Dimer-Concentration to Critical Transfer Concentration, Concentrations of Monomer $C_{m}$ and Total Dimer $C_{d}$, and Apparent Critical Distance $R_{o}$ of Energy Transfer

\begin{tabular}{|c|c|c|c|c|c|c|c|}
\hline $\begin{array}{c}\text { Total } \\
\text { Concentration } \\
\text { C }[M]\end{array}$ & A1 & A2 & $\begin{array}{c}\text { Monomer } \\
\text { Concentration } \\
C_{m}(M)\end{array}$ & $\begin{array}{c}\text { Total Dimer } \\
\text { Concentration } \\
C_{d}[M !\end{array}$ & $=c_{d:}^{Y} / c_{0}$ & $\begin{array}{c}\text { Apparent Critical } \\
\text { Distance Cubed } \\
R_{0}^{3}\left[R^{3}\right]\end{array}$ & $\begin{array}{c}\text { Apparent Critical } \\
\text { Distance } \\
\mathbb{R}_{0}[\& 1\end{array}$ \\
\hline $8.4 \times 10^{-3}$ & 0.48 & 0.52 & $2.0 \times 10^{-4}$ & $4.1 \times 10^{-3}$ & 0.38 & $3.7 \times 10^{4}$ & 33 \\
\hline $8.4 \times 10^{-4}$ & 0.26 & 0.74 & $5.2 \times 10^{-5}$ & $3.9 \times 10^{-4}$ & 0.12 & $1.2 \times 10^{5}$ & 50 \\
\hline $8.4 \times 10^{-5}$ & 0.07 & 0.93 & $2.0 \times 10^{-5}$ & $3.2 \times 10^{-5}$ & 0.03 & $4.2 \times 10^{5}$ & 75 \\
\hline $8.4 \times 10^{-5}$ & 0.13 & 0.87 & $1.1 \times 10^{-6}$ & $3.6 \times 10^{-6}$ & 0.00 & - & $\ldots$ \\
\hline
\end{tabular}

where the first and second terms describe the fast fluorescence decay of the $Y$-band, and the slow and dynamically quenched fluorescence decay of the usual band, respectively. As a result of numerical fitting of (1) to the measured total fluorescence decays at different concentrations in Fig. $4, \tau_{1}, \tau_{2}, A_{1}, A_{2}$, and $\gamma$ are determined and given in Table I. The solid lines in Fig. 4 represent the numerical fitting curves. The fluorescence lifetimes of the $Y$-band from the $D$ aggregate and $S$ dimer, and of the usual band from the monomer are $120 \mathrm{ps}$ and $3.6 \mathrm{~ns}$, respectively. As the HPD concentration decreases, $A_{1}$ proportional to the sum concentration of the $D$ aggregate and the $S$ dimer decreases, while $A_{2}$ proportional to the concentration of the monomer increases.

For simplification of the discussion, we assume as follows: 1 ) all the $D$ aggregates are only the $E$ dimer, 2) the molar extinction coefficients $\epsilon_{1}$ at the wavelength $(570 \mathrm{~nm})$ of the excitation pulse, the fluorescence quantum yields $\phi_{1}$, and the fluorescence lifetimes $\tau_{1}$ of the $E$ dimer and those of the $S$ dimer are the same. The assumptions allow us to express the ratio of $A_{2} / A_{1}$ as

$$
\frac{A_{2}}{A_{1}}=\frac{C_{m} \epsilon_{2} \phi_{2} / \tau_{2}}{C_{d} \epsilon_{1} \phi_{1} / \tau_{1}}
$$

where $C_{m}, \epsilon_{2}$ and $\phi_{2}$ are the concentration, the molar extinction coefficient at $570 \mathrm{~nm}$, and the fluorescence quantum yield of the monomer respectively, and $C_{d}$ is the total dimer concentration of the $E$ and $S$ dimers. By using the obtained values of $\tau_{1}, \tau_{2}, A_{1}$, and $A_{2}$, and the ratio values of $\epsilon_{2} / \epsilon_{1}=$ 0.65 (from the absorption spectra in the previous section), and $\phi_{2} / \phi_{1}=1000$ (the assumed value) in (2), the monomer concentration and the total dimer concentration for different HPD concentrations are calculated as given in Table I. According to a theory of the Förster-type energy transfer between donor and acceptor molecules [20]- [21], the $\gamma$ in (1) is equal to the ratio $C_{d} / C_{o}$ of the acceptor concentration to the critical transfer concentration. For the HPD solution, the acceptor concentration is equal to the total dimer concentration. The critical transfer concentration is expressed by $\left(7.35 / R_{0}\right)^{3}$ with the critical transfer distance $R_{o}$ units of $\AA$ [21]. The $R_{o}$ is used to indicate the distance at which energy transfer has the same probability as the sum of all other excited state deactiva-

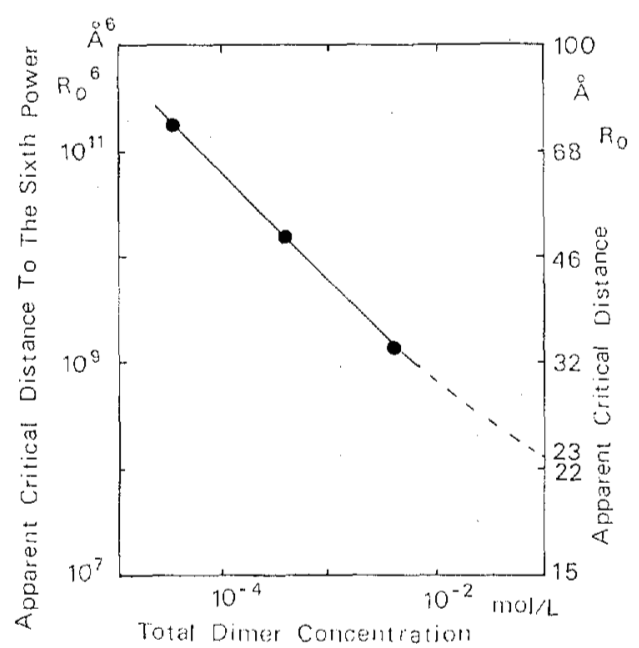

Fig. 8. Total dimer-concentration dependence of the apparent critical distance $R_{O}$ of energy transfer for the HPD solution.

tion processes of the monomer. The distance is given by

$$
R_{o}^{6}=\frac{(9000 \ln 10) k^{2} \phi_{2}}{128 \pi^{5} n^{4} N} \int_{0}^{\infty} \frac{f_{2}(\nu) \epsilon_{1}(\nu)}{\nu^{4}} d \nu
$$

where $k^{2}$ is an orientation factor, $\left(k^{2}=\frac{2}{3}\right.$ for HPD with a random direction distribution in the aqueous solution [22]), $\phi_{2}$ is the fluorescence quantum yield of the monomer in the absence of the $E$ and $S$ dimers, $n$ is the refractive index of the solvent, $N$ is Avogadro number, $f_{2}(\nu)$ and $\epsilon_{1}(\nu)$ are the spectral distribution of the monomer fluorescence and the molar extinction coefficient of the $E$ and $S$ dimers respectively, and $\nu$ is the wavenumber [20]-[21].

The combination of the toal dimer concentration $C_{d}$ with the $\gamma$ enables us to determine the apparent critical distance $R_{o}$, and the result is also given in Table I. The dependence of the apparent critical distance on the total dimer concentration is shown in Fig. 8. The distance is rapidly decreasing with the increase of the total dimer concentration, like the experimental results of the resonance transfer from rhodamine $B$ monomer to dimer [19] and from DODCI to malachite green and to DQOCI [23]. The true critical distance $R_{o t}$ is determined by extrapolating the total dimer concentration toward the higher 


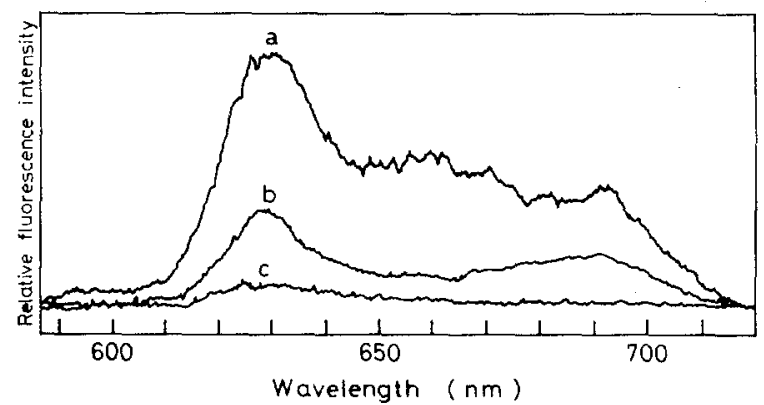

Fig. 9. Static fluorescence spectra from (a) human gastric cancers, (b) the erosion of the cancers, and (c) the surrounding normal cells at the early stage in vivo $48 \mathrm{~h}$ after HPD injection.

concentration and is estimated to be $23 \AA$. This value is almost equal to the value of $24 \AA$ which is evaluated by using (3) on the basis of data of the spectral overlap in Fig. 3 .

Finally, we describe the correlation between the picosecond time-resolved fluorescence spectra from the HPD solution and the static fluorescence spectra from HPD in cancerous cells in vivo. The static fluorescence spectra from the human gastric cancers, the erosion of the cancers and the surrounding normal cells at the early stage ( 72 years old male) after injection of the HPD solution ( $5 \mathrm{mg} / \mathrm{kg}$ weight) were simultaneously measured by using a fiber endoscope with a Jobin Yvon HR320 polychromator and a Princeton Applied Research OMA I under $405 \mathrm{~nm} \mathrm{Kr}$-ion laser pumping. The results measured in $48 \mathrm{~h}$ after injection showed that only the static fluorescence spectra from the gastric cancers have a third emission band which corresponds to the $Y$-band as shown in Fig. 9. It was also observed that the third emission band gradually appears at only the gastric cancers as time passes after injection. Therefore, it seems that the head-to-tail aggregates emitting the $Y$-band play a role important for the selective accumulation of HPD in cancerous cells, and the fluorescence lifetime of the aggregates is remarkably increased by binding to some biomolecule in vivo. As for this point, further investigation is needed.

\section{CONCLUSION}

From the detailed measurement of absorption spectra and excitation spectra for the HPD solution, it has been found that the HPD solution at high concentration contains the $D$ aggregate including mainly the $E$ dimer and the $S$ dimer, and both the absorption spectra are similar. The measurement of the picosecond time-resolved fluorescence spectra by means of the two-dimensional synchroscan streak camera has shown that a new fast emission band due to the head-to-tail $D$ aggregate and $S$ dimer exists. The mechanism of the fluorescence relaxation in the HPD solution has been made clear. That is, the fluorescence decays are due to the fast emission $\left(\tau_{1}=120 \mathrm{ps}\right)$ from the head-to-tail $D$ aggregate and $S$ dimer and the slow emission $\left(\tau_{2}=3.6 \mathrm{~ns}\right)$ from the monomer. The latter emission is quenched by Förster-type resonance energy transfer from the monomer to the $D$ aggregate and $S$ dimer. Furthermore, from the observation of an emission band corresponding to the fast emission band only at the gastric cancers in vivo, it has been suggested that the head-to-tail $D$ aggregate and $S$ dimer has a important role for the selective accumulation properties of the HPD solution.

\section{ACKNOWLEDGMENT}

The authors would like to thank S. Tanaka for help with the computer analysis.

\section{REFERENCES}

[1] T. J. Dougherty, J. E. Kaufman, A. Goldfarb, K. R. Weishaupt, D. G. Boyle, and A. Mittelman, "Photoradiation therapy for the treatment of malignant tumors," Cancer Res., vol. 38, p. 2628, 1978.

[2] T. J. Dougherty and R. E. Thoma, Lasers in Photomedicine and Photobiology, R. Pratesi and C. A. Sacchi, Eds, Berlin, Germany: Springer-Verlag, 1980, p. 67.

[3] Y. Hayata, H. Kato, C. Konaka, J. Ono, and N. Takizawa, "Hematoporphyrin derivative and laser photoradiation in the treatment of lung cancer," Chest., vol. 81, p. 269, 1982; and private communications.

[4] See, for example, Tech. Dig. Clyton Found. Symp. Porphyrin Localization Treatment Tumors, Santa Barbara, CA, Apr. 24-28, 1983.

[5] T. J. Dougherty, D. G. Boyle, K. R. Weishaupt, B. A. Henderson, W. R. Potter, D. A. Bellnier, and K. E. Wityk, "Photoradiation therapy-clinical and drag advances," in Proc. Work. Porphyrin Sensitization, Washington, D.C., Sept. 1981.

[6] W. I. White, The Porphyrins, vol. V, D. Dolphin, Ed. New York: Academic, 1979, pp. 303-339.

[7] W. A. Gallagher and W. B. Elliott, Ann. N. Y. Acad. Sci., vol. 206, p. $463,1973$.

[8] S. B. Brown and M. Shillcock, "Equilibrium and kinetic studies of the aggregation of porphyrins in aqueous solution," Biochem. J., vol. 153, p. $279,1976$.

[9] G. A. Karns, W. A. Gallagher, and W. B. Elliott, "Dimerization constant of water-soluble porphyrins in aqueous alkali," Bioorg. Chem., vol. 8, p. 69, 1979.

[10] R. Margalit, N. Shakla, and S. Cohen, "Fluorimetric studies on the dimerization equilibrium of protoporphyrin IX and its haemato derivative," vol. 209 , p. $547,1983$.

[11] J. Moan and S. Sommer, Photobiochem., Photobiophys., "Fluorescence and absorption properties of the components of hematoporphyrin derivative," vol. 3 , p. 93, 1981.

[12] T. J. Dougherty, Cancer Res., vol. 42, p. 1188, 1982; see also, Photochem., Photobiol., vol. 38, p. 377,1983 ; and private communications.

[13] A. Andreoni, R. Cubeddu, S. De Silvestri, P. Laporta, G. Jori, and E. Reddi, Chem. Phys. Lett., vol. 88, p. 33, 1982.

[14] M. Yamashita, T. Sato, K. Aizawa, and H. Kato, "Picosecond fluorescence spectroscopy of hematoporphyrin derivative and related porphyrins," Picosecond Phenomena III, K. B. Eisenthal, R. M. Hochstrasser, W. Kaiser, and A. Laubereau, Eds. Berlin, Germany: Springer-Verlag, 1982, p. 298.

[15] C. Hanzlik, W. Knox, T. Nordlund, R. Hilf, and S. Gibson, "Picosecond fluorescence of hematoporphyrin derivative and its components in aqueous solution," in Tech. Dig. Clyton Found. Symp. Porphyrin Localization Treatment Tumors, Santa Barbara, CA, Apr. 24-28, 1983, Preclin. Sess. I-3, also see Dept. Physics, Astronomy, Univ. Rochester, Rochester, NY, Biolog. Phys., Group, Tech. Rep. 37, May 2, 1983.

[16] M. Kasha, "Energy transfer mechanisms and the molecular exciton model for molecular aggregates," Radia. Res., vol. 20, p. 55, 1963; see also, M. Kasha, H. R. Rawls, and M. A. El-Bayoumi, in Proc. 8th Euro. Congr. Molec. Spectroscopy. London, England: Butterworths, 1965, pp. 371-392.

[17] V. Sundström and T. Gillbro, "Radiationless processes in aggregated chromophores of the dye diethylthiadicarbocyanineiodide in aqueous solutions," in Tech. Dig. Ultrafast Phenomena, Monterey, CA, June 1984, p. ThE7.

[18] M. Yamashita, T. Honda, T. Sato, and K. Aizawa, "A compact synchroscan streak camera using a microchannel plate incorporated tube," IEEE Trans. Instrum. Meas., vol. IM-32, p. 124, 1983.

[19] N. Nakashima and K. Yoshihara, "Time-resolved measurements of electron and energy transfer of rhodamine B monolayer on the surface of organic crystal," J. Chem. Phys., vol. 73, p. 3553 , 1980.

[20] Th. Förster, Ann. Phys., vol. 2, p. 55, 1948; see also, Z. Naturforsch., vol. A4, p. 321, 1949.

[21] N. Mataga and T. Kubota, Molecular Interactions and Electronic Spectra. New York: Marcel Dekker, 1970, p. 171. 
[22] M. Z. Maksimov and I. M. Rozman, "On energy transfer in solid solution," Opt., Spectro., vol. 10, p. 337, 1961.

[23] M. C. Adams, D. J. Bradley, W. Sibbett, and J. R. Taylor, "Realtime picosecond measurements of electronic energy transfer from DODCI to malachite green and DQOCI," Chem. Phys. Lett., vol. 66, p. $428,1979$.

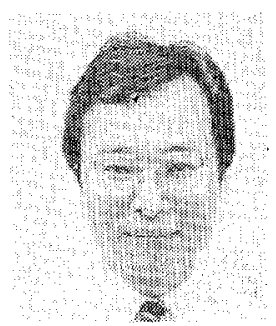

Mikio Yamashita was born in Kyoto, Japan, on February 15, 1946. He received the M.S. and $\mathrm{Ph} . \mathrm{D}$. degrees in electronics engineering from Kyoto University, Kyoto, Japan, in 1970 and 1976, respectively.

Since joining the Laser Research Section, Radio- and Opto-Electronics Division, Electrotechnical Laboratory, Tokyo, Japan, in 1970 , he has been engaged with laser excitation ESR studies on laser dyes, development of the tunable dye laser, and its application to the isotope separation. In 1978 he was a Visiting Research Associate at Imperial College. His research interests are currently in the generation of the ultrashort pulse and its application including photochemical biotechnology.

Dr. Yamashita is a member of the Physical Society of Japan, the Japan Society of Applied Physics, and the Laser Society of Japan.

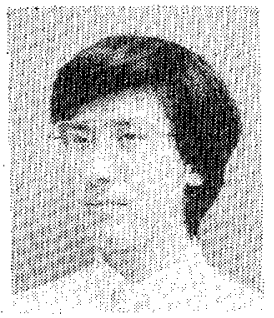

Masahide Nomura was born in Miyagi, Japan, on April 19, 1960. He received the B.S. degree in physics from Tohoku University, Sendai, Japan, in 1983 . He is currently working towards the M.S. degree in biophysics at Tohoku University.

Since August 1983, he has been working with Dr. M. Yamashita et al. in the field of picosecond spectroscopy at Electrotechnical Laboratory belonging to MITI.

Mr. Nomura is a member of the Japan Society of Applied Physics.

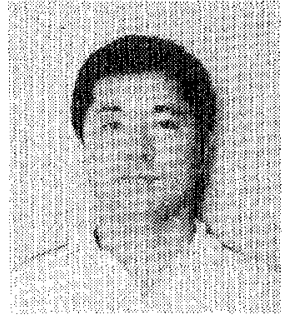

organic materials.

Dr. Kabayashi is a member of the Chemical Society of Japan, the Physical Society of Japan, and the Japan Society of Applied Physics.

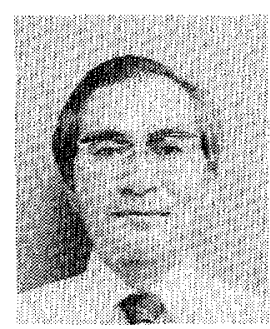

Takuzo Sato was born in Akita, Japan, on October 27, 1940. He received the M.S. and Ph.D. degrees in electronics engineering from Tohoku University, Sendai, Japan, in 1965 and 1980 , respectively.

In 1965 he joined the Electrotechnical Laboratory, Tokyo, Japan, where he has been engaged in research on the $Q$-switched ruby laser, dye lasers, mixing of laser beams, laser radar, and excimer lasers. His fields of interest are high-power lasers and their applications.

Dr. Sato is a member of the Institute of Electronics and Communication Engineers of Japan, the Japan Society of Applied Physics, and the Laser Society of Japan.

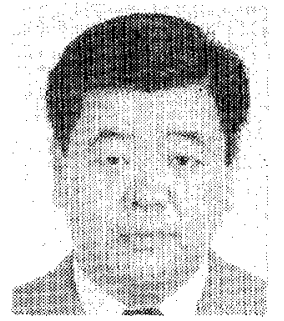

Katsuo Aizawa was botn in Yokohama, Japan, on September 5, 1939. He received the Ph.D. degree in medicine from Tokyo Medical College Tokyo, Japan, in 1971.

Currently, he is an Associate Professor with the Department of Physiology, Tokyo Medical College. He has been engaged in the laser excitation Raman spectrum studies on abdominal nerve cord membrane and some biological materials.

Dr. Aizawa is a member of the Physiological Society of Japan, the Biophysical Society of Japan, the Japan Society for Cancer Therapy, the Cancer Society of Japan, and the Japan Society for Laser Medicine. 